\title{
Experimental Investigation of the Effects of Arterial Geometries in Different Severities of Symmetrical Stenosis on Pressure Drop and Pump Power
}

Fatin SONMEZ ( $\nabla$ fatinsonmez@artvin.edu.tr)

Artvin Coruh University

Orhan YILDIRIM

Ataturk University

Sendogan KARAGOZ

Ataturk University

Fuat GUNDOGDU

Ataturk University

Research Article

Keywords: Peristaltic Pump, Heart, Power, Stenosis, Pulse, Pressure Drop

Posted Date: July 28th, 2021

DOI: https://doi.org/10.21203/rs.3.rs-753411/v1

License: (c) (i) This work is licensed under a Creative Commons Attribution 4.0 International License.

Read Full License 


\title{
EXPERIMENTAL INVESTIGATION OF THE EFFECTS OF ARTERIAL GEOMETRIES IN DIFFERENT SEVERITIES OF SYMMETRICAL STENOSIS ON PRESSURE DROP AND PUMP POWER
}

\author{
Fatin SONMEZ ${ }^{* 1}$, Orhan YILDIRIM ${ }^{2}$, Sendogan KARAGOZ ${ }^{2}$ Fuat GUNDOGDU $^{3}$ \\ * Artvin Vocational School, Artvin Coruh University, Artvin, Turkey \\ ${ }^{2}$ Department of Mechanical Engineering, Ataturk University, Erzurum, Turkey \\ ${ }^{3}$ Faculty of Medicine, Ataturk University, Erzurum, Turkey \\ *Corresponding Author: Fatin SONMEZ; E-mail: fatinsonmez@artvin.edu.tr
}

\begin{abstract}
Biomedical studies is among the multidisciplinary studies attracting most interest in recent years. Blood and vessel interactions and consequent hemodynamic effects cause cardiovascular diseases. A testing setup constituted by a peristaltic pump (similar to the heart mechanism) system was installed. The purpose of the experimental study presented is to investigate the effect, pressure drop, peristaltic pump inlet and outlet pressure and most importantly, the amount of power consumed by the peristaltic pump regarding arterial stenosis severity with varying areal stenosis percentages. The tests were performed for the pulse values from 54 to $168 \mathrm{bpm}$ by setting up models with $0 \%, 60 \%, 70 \%$ and $80 \%$ symmetrical stenosis severities.

In the study, the pressure difference in the test area increased concomitantly with elevated pulse value and increased stenosis severity. This situation revealed that as the intensity of narrowing increases in vessels, the narrowing space differential pressure increases, and this amount increases even more with increased exertion. The pressure at the peristaltic pump outlet increased concomitantly with elevated pulse value and increased stenosis severity. The peristaltic pump overworked to overcome the increased differential pressure related to the increased pulse value and stenosis severity. This result of the experimental data reveals the necessity to avoid activities requiring high pulse in human arteries similarly with a high percentage of stenosis.
\end{abstract}

Keywords: Peristaltic Pump, Heart, Power, Stenosis, Pulse, Pressure Drop

\section{Introduction}

In recent years, multidisciplinary studies have become prominent due to advances in all fields of science. Biomedical research is one of these multidisciplinary studies. Biomedical research presumes that cardiovascular diseases are directly affected by the structure of the heart, blood forces and pressure on vessels. Additionally, scientists work collaboratively to find new relationships in order to explore these effects in depth. 
Cardiovascular diseases are a group of disorders of blood vessels and the heart. It is the number one cause of disease-related deaths, causing 17.3 million deaths across the World, and this number is expected to rise to a 23.6 million by the year 2030 [1,2]. While cardiovascular diseases paint such a picture in the globe, the Europe is also subject to a similar situation as they are the leading cause of death every year [3]. Among the cardiovascular diseases, particularly atherosclerosis is the leading cause of death in developed countries [4,5]. There are a limitless number of physiological and biological causes which result in occurrence and development of atherosclerosis. Blood flow is one of the physiological causes. Arterial stenosis is the narrowing of the blood artery caused by the thickening of the vessel lumen due to atherosclerotic plaque buildup. In stenotic arteries, the diameter of blood vessels is reduced due to cholesterol accumulation and lipid deposition near the artery walls $[6,7]$.

Experimental and quantitative research studies on the cardiovascular system have been conducted in recent years. It has been observed that while some of these are studies related to narrowed vessel geometries, most are quantitative research conducted in order to comprehend the hemodynamic structure of blood flow in the vessel. In preliminary studies of stenotic arteries, most researchers regarded human blood as a Newtonian fluid and considered stenosis as a symmetrical narrowing to simplify the problem [8-10].

Some researchers regarded it as a non-Newtonian fluid for a more detailed modelling of blood flow in stenotic arteries. In their study, Jung et al. [11] numerically analyzed the flow of nonNewtonian fluid under pulsatile flow conditions in a symmetrical rigid artery with a trapezoidal constriction profile utilizing the finite volume method. In the study they conducted, Roy et al. [12] investigated the velocity and pressure distributions of the blood flow depending on various stenosis severities of a stenotic artery by modelling them with the non-Newtonian flow model Carreau-Yasuda in CFD (Computational Fluid Dynamics).

Some researchers compared blood flow for the Newtonian and non-Newtonian cases $[13,14]$. The experimental study conducted by Anastasiou et al. [15] predicted the change during wall shear stress and pulsatile flow with in vivo study under pulsatile flow conditions simulating a bifurcated small artery. The numerical study conducted by Husain et al. [16] investigated the blood flow in hardened stenotic arteries with four non-Newtonian models at three different intensities of stenosis (20\%, 50\% and 80\%). Apostolidis et al. [17] assessed the effects of non-Newtonian blood rheology with arterial flow simulations in their numerical study. They achieved this by comparing a) the Casson viscoplastic model, which is a non-Newtonian flow model, and b) the converging solutions obtained from the Newtonian flow model.

Some researchers investigated the narrowing occurrences in a stenotic artery as an asymmetrical model. In the experimental study they conducted, Back et al. [18] performed pressure measurements of the Re number between the values of 60 and 500 on a Cx coronary artery removed from a cadaver with a vascular disorder. Vesely et al. [19] experimentally studied the pressure loss coefficient for 
varying stenosis eccentricity and shape. Novakova et al. [20] examined the effect of stenosis severity and eccentricity on pressure drop in their experimental study. Kamandar et al. [21] analyzed the blood flow behavior and the severity of arterial occlusion based on different geometrical shapes of stenosis such as elliptical, trapezoidal and triangular.

The cardiovascular system is governed by pulsatile blood flow. Some researchers have studied flow as pulsatile as in human physiology [22,23]. In their numerical study, Rabby et al. [24] examined the flow in the non-symmetrical stenosis tube, by modelling it as two-dimensional under pulsatile and non-Newtonian flow conditions based on the finite volume method. Beratlis et al. [25] carried out a numerical and experimental research to study the pulsatile and Newtonian flow in a rectangular section stenotic with a $50 \%$.

This study aimed to investigate the effects of different rates of stenosis in a human vessel on some hemodynamic properties and the peristaltic pump (heart). The study was carried out experimentally. Even though a significant number of studies have been conducted to investigate blood flow in stenotic arteries in recent years, not many studies have been reported on blood flow in stenoses of different shapes and sizes in arteries. Therefore, this study was motivated to investigate the effect of different stenotic space percentages on blood flow. Since no studies researching into the impact of hemodynamic effects on the heart depending on the constriction geometry in the literature, the originality of this study stands out at this point.

\section{Experimental Setup and Test Procedure}

The purpose of this experimental study is to determine the effect of various severities of arterial stenosis on pressure drop, pump outlet pressure and the amount of power consumed by the pump. The materials used in the setup of the test system whose schematic diagram is demonstrated in Figure 1 and their functions can be summarized as follows: In the system, a $30 \mathrm{~cm}$ silicone hose of $8 \mathrm{~mm}$ diameter and 40-80 Shore-A hardness value was utilized in the test area. A glass pool is constructed to keep the temperature of the work area at a constant $37{ }^{\circ} \mathrm{C}$. The heated bath is utilized in the experimental system for two purposes. The first was to keep the temperature of the test pipe with a narrowed geometry constant at $37{ }^{\circ} \mathrm{C}$ by keeping the water in the glass pool constant at this temperature. The second is to keep the temperature of the operation fluid inside the heat exchanger placed in the heated bath also constant at $37{ }^{\circ} \mathrm{C}$ (Daihan Scientific, MaXircu CR-8). A differential pressure transmitter (SHHDP-9600) is utilized to measure the pressure difference at the inlet and outlet of the test area in the system. An electromagnetic flowmeter is integrated into the system to measure the flow of the operation fluid in the system (Euromag, MC308C, MUT500EL). The data received from the thermocouples, electromagnetic flow meter and pressure transmitter are transferred to the computer via a data reading card (Novus, Field logger) and also analyzed through the system. 3 thermocouples are used on the test pipe. A peristaltic pump with a maximum flow rate of $593 \mathrm{l} / \mathrm{h}$ and maximum pressure of 12 bar is utilized in the system to facilitate the circulation of the operation fluid 
(Verderflex, Dura15). An inverter is employed to ensure operation of the peristaltic pump at desired speeds by regulating its operating frequency (Invt, G10).

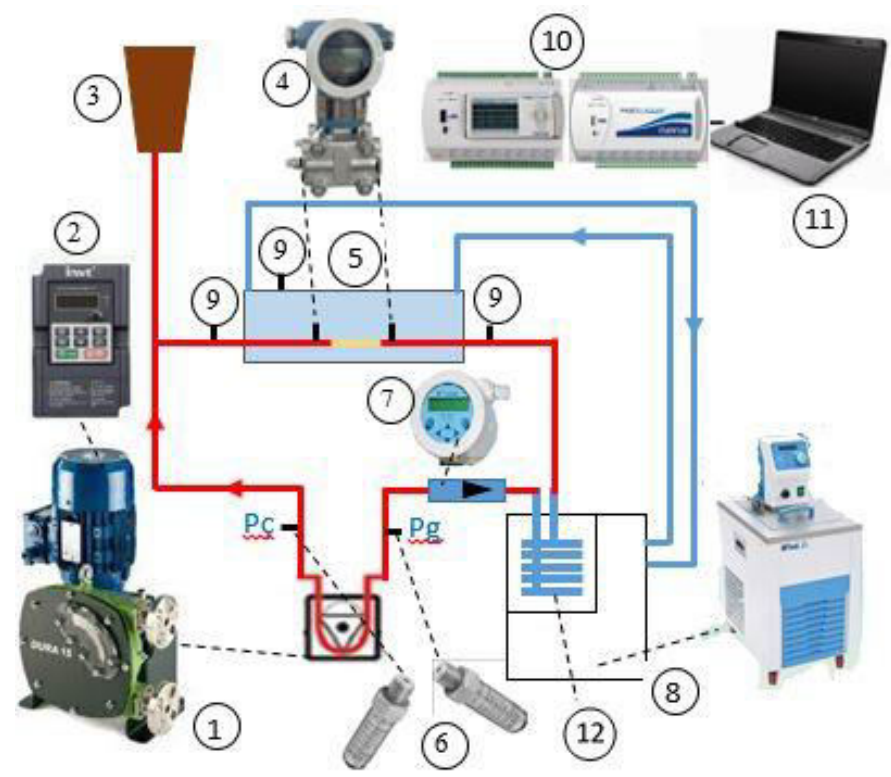

(1) Peristaltic pump

(2) Inverter

(3) Reservoir

(4) Differential pressure transmitter

(5) Test area

(6) Piezoresistive pressure transmitter

(7) Flow meter

(8) Heated bath

(9) Thermocouple

(10) Data logger

(11) Computer

(12) Spiral heat exchanger

Figure 1. Schematic diagram of the test system

In order measure the inlet and outlet pressure of the peristaltic pump, one piezoresistive pressure transmitter for each was used (Dixell, PA-21SC).

The study experimentally investigated the pressure drop, peristaltic pump outlet pressure and power consumption of the pump regarding various stenotic models. An $8 \mathrm{~mm}$ diameter $300 \mathrm{~mm}$ long flexible hose constitutes the test area. Stenosis models were drawn in a solid modeling program and produced in a 3D printer, and their areal rates of stenosis were set to $0 \%, 60 \%, 70 \%$ and $80 \%$ (Fig. 2).

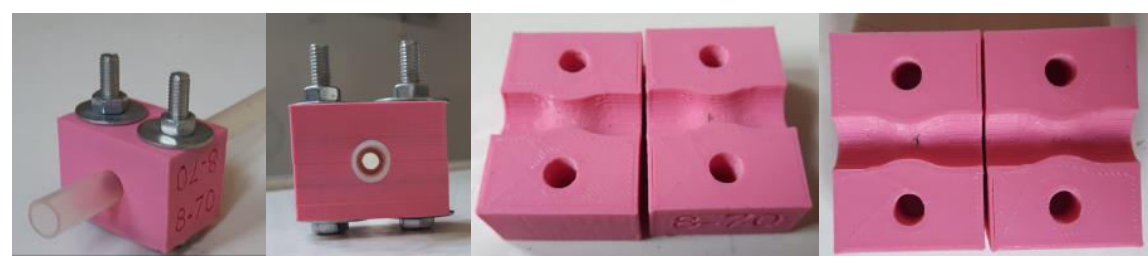

Figure 2. The mold with narrowed geometry produced in 3D printer

Pure water was used as the operation fluid in the study. Blood is a non-Newtonian fluid and its viscosity changes with its shear rate. It exhibits Newtonian flow properties at high shear rates. NonNewtonian behavior is most noticeable in small branches and capillaries. In most arteries, blood behaves in a Newtonian flow fashion and the viscosity can be taken as constant $[26,27]$. Therefore, the effect of non-Newtonian properties of blood is considered minimal on the flow velocities and vessel size $(8 \mathrm{~mm})$ adopted in these tests.

In the experimental study, the following operations were performed respectively in order to make the necessary measurements on the test pipe: Firstly, the pure water utilized as the operation fluid was filled into the entire system and purified from air. The pure water level in the reservoir was 
constantly checked. In order to determine the speed of the fluid in the system, the frequency values corresponding to the pulse values that will change the revolutions of the peristaltic pump were adjusted through the inverter. In this way, the speed of the operation fluid was adjusted by changing the number of revolutions of the peristaltic pump. The frequency values set manually on the inverter range between 20 and $60 \mathrm{~Hz}$, and the pulse values corresponding to these values are demonstrated in Table 1. Pulse refers to the number of heart contractions per minute, and its unit is bpm. The work performance of the heart varies over a wide range of activities from the sleeping state to the state during physical activities such as sports. In the experimental system, this similarity was achieved in different stenosis severities by adjusting the peristaltic pump frequency.

Table 1. Pulse values corresponding to frequency

\begin{tabular}{|c|c|}
\hline Frequency [Hz] & Pulse (bpm) \\
\hline 20 & 54 \\
\hline 30 & 84 \\
\hline 40 & 114 \\
\hline 50 & 132 \\
\hline 60 & 168 \\
\hline
\end{tabular}

The pressure difference in the pump was calculated by measuring the pressures at the inlet and outlet of the peristaltic pump. The power consumed by the pump based on the pulse values and the stenosis severity was acquired by multiplying the $\mathrm{P}_{\text {out }}-\mathrm{P}_{\text {in }}$ pressure difference with the flow rate [28].

$$
\dot{W}=2,78 * 10^{-5} * \dot{V} \cdot\left(P_{\text {out }}-P_{\text {in }}\right)
$$

Here, $\dot{W}$ is the power (Watt) consumed by the peristaltic pump, $\dot{V}$ is the volumetric flow $(1 / \mathrm{h})$, $P_{\text {in }}$ is the peristaltic pump inlet pressure (mbar), and $P_{\text {out }}$ is the peristaltic pump outlet pressure (mbar).

Pressure transmitter outputs were sampled on the data reading card, and data was obtained. A U-manometer was employed to calibrate the transmitters. Before each test, the pressure transmitters were reset and the pump was adjusted to ensure an accurate flow velocity. Healthy human body temperature is approximately $37{ }^{\circ} \mathrm{C}$. The heated bath is adjusted to $37{ }^{\circ} \mathrm{C}$ in order to bring the operation fluid to this temperature. Before recording the test data, the system was run for approximately 30 minutes until it became steady. After the system remained in the steady-state, the required measurement value was taken.

\section{Findings and Discussion}

The research was analyzed with the help of the charts generated based on the data obtained from the experiments. Pressure drop $(\Delta \mathrm{P})$, flow rate, $\mathrm{P}_{\text {out }}, \mathrm{P}_{\text {out }}-\mathrm{P}_{\text {in }}$ and $\dot{W}$ values depending on the varying stenosis severities at varying pulse values from 54 to $168 \mathrm{bpm}$ were established with the data acquired. 
Furthermore, pump outlet pressure amplitudes were established by taking the 60 -second $\mathrm{P}_{\text {out }}$ values at varying stenosis severities.

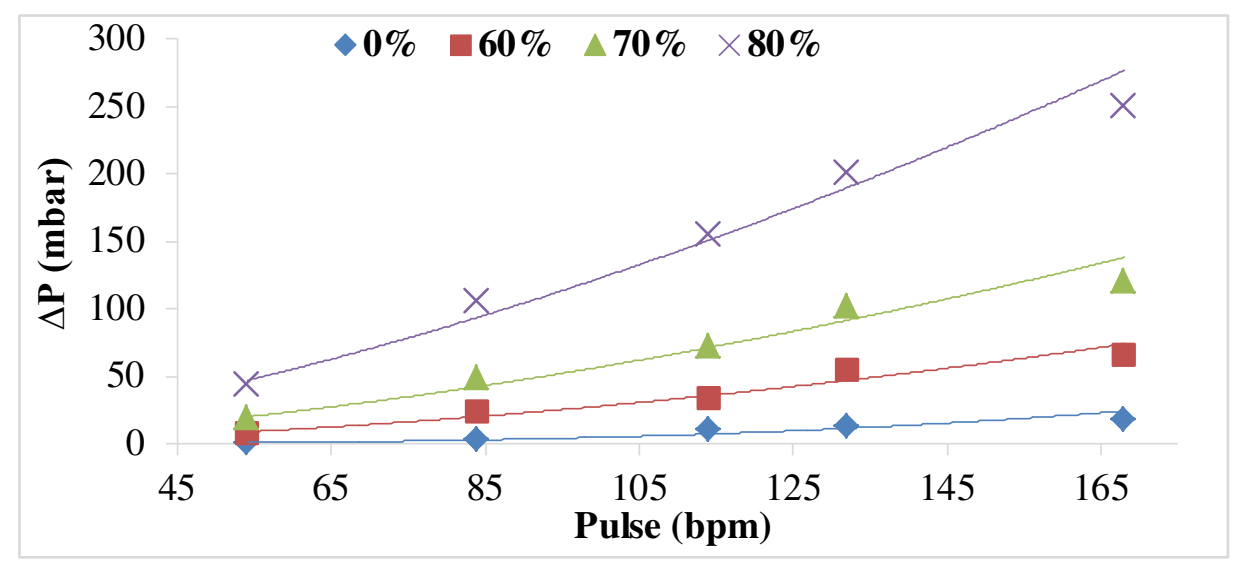

Figure 3. The effect of pulse values at different stenosis severities on pressure drop

In Figure 3, the effect of the silicon pipe with areal rates of symmetrical stenosis at $0 \%, 60 \%$, $70 \%$ and $80 \%$ on $\Delta \mathrm{P}$ based on the pulse values of the test area of its location were exhibited. With the increasing pulse values, $\Delta \mathrm{P}$ in the test area elevates [29]. However, comparing the stenosis severities within the same pulse values revealed that the $\Delta \mathrm{P}$ value increased as the rate of narrowing increased. In addition, while $\Delta \mathrm{P}$ values are close to each other at different stenosis severities for low pulse values, the $\Delta \mathrm{P}$ difference between these values increases with increasing pulse values. This indicates that as the severity of stenosis increases in vessels, the difference increases between the amount of pressure in front of and behind the stenosis space, and this amount escalates even more with increasing effort [30].

In Figure 4, the effect of the flow amount in the test area of the pipe with areal rates of symmetrical stenosis at $0 \%, 60 \%, 70 \%$ and $80 \%$ on $\Delta \mathrm{P}$ is exhibited. With increasing flow rate, the $\Delta \mathrm{P}$ in the test area increases [19]. However, comparing the stenosis severities within the same pulse values revealed that the $\Delta \mathrm{P}$ value increased as the rate of narrowing increased. Additionally, while $\Delta \mathrm{P}$ values

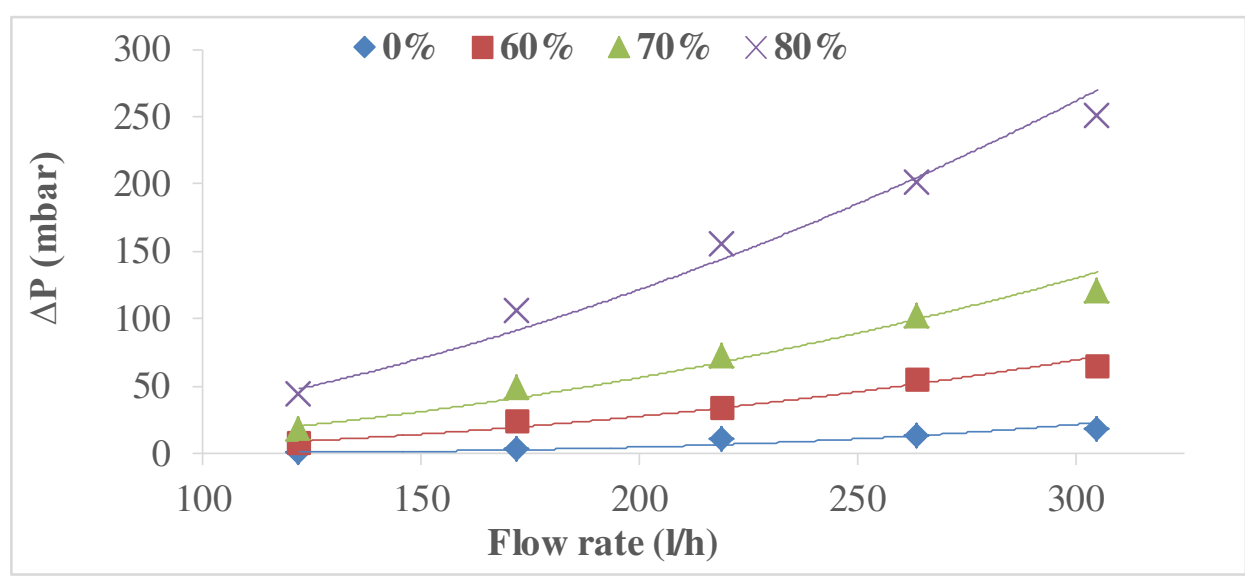

Figure 4. The effect of the flow rate on pressure drop in different stenosis severities 
are close to each other at different stenosis severities for low flow rates, the $\Delta \mathrm{P}$ difference between these values grows concomitantly with the increasing flow rate. This concludes that as the stenosis severity in the vessels increases, the difference between the $\Delta \mathrm{P}$ values caused by the stenosis increases, and this amount increases even more with increasing pulse value [20].

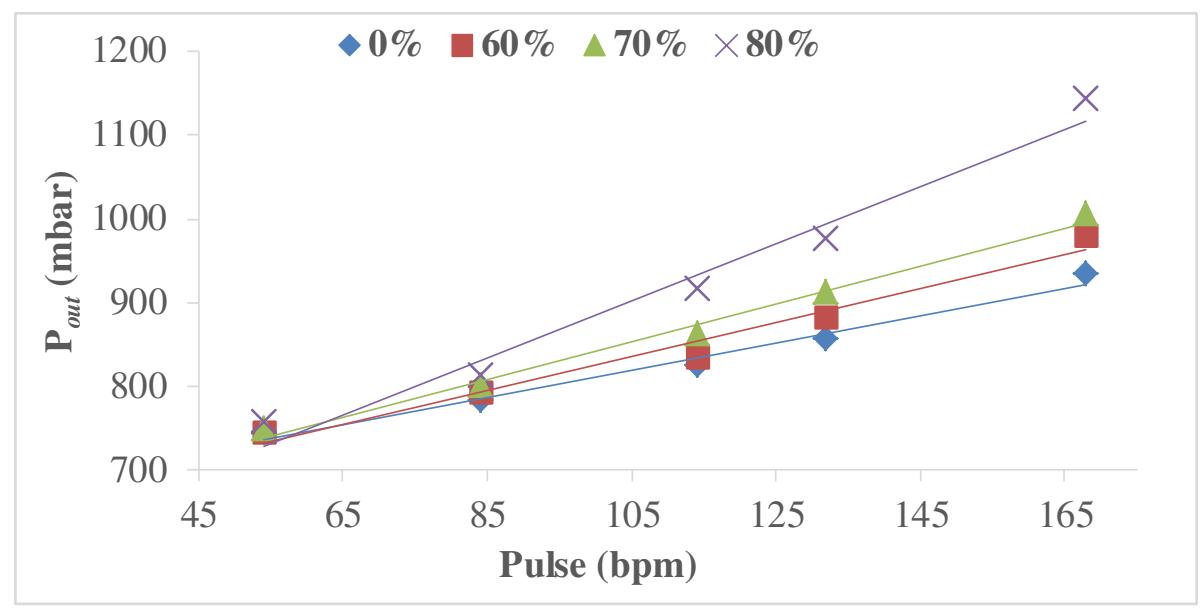

Figure 5. The effect of pulse values at different stenosis severities on the pump outlet pressure

In Figure 5, the effect of the pulse values in the peristaltic pump of the test area silicon pipe with areal rates of symmetrical stenosis at $0 \%, 60 \%, 70 \%$ and $80 \%$ on the pump outlet pressure is demonstrated. In the figure, the pressure at the peristaltic pump outlet increases with increasing pulse values. Pressure increase is expected at the pump outlet concomitantly with the increasing pump flow rate and accordingly the pulse values [28]. Furthermore, a gradual increase is observed in pump outlet pressure with the increase in pulse values ranging from a healthy artery with a $0 \%$ stenosis rate to an artery with a stenosis rate of $80 \%[30,31]$.

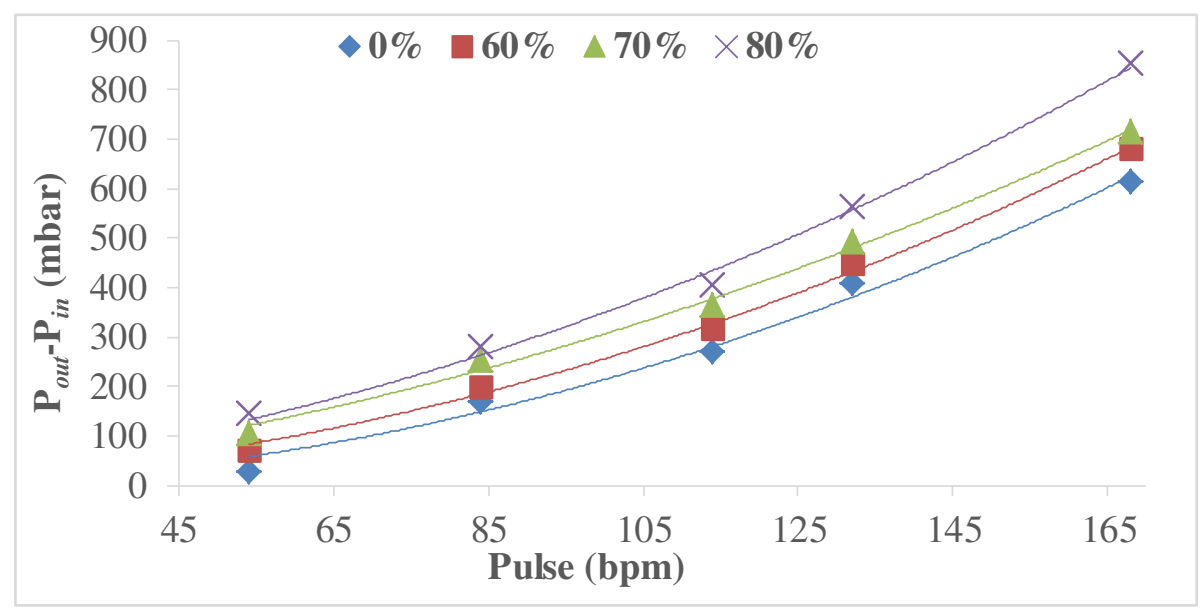

Figure 6. The effect of pulse values at different stenosis severities on differential pressure of pump

In Figure 6, the effect of the pulse values in the peristaltic pump of the test area silicon pipe with areal rates of symmetrical stenosis at $0 \%, 60 \%, 70 \%$ and $80 \%$ on the differential pressure of the pump is exhibited. In the figure, the differential pressure at the peristaltic pump outlet increases with the 
increasing pulse values. In other words, the differential pressure in the pump increases with the increasing pump flow rate and accordingly the pulse values [32]. Additionally, the increase in the stenosis severity for the same pulse values leads to more differential pressure in the peristaltic pump.

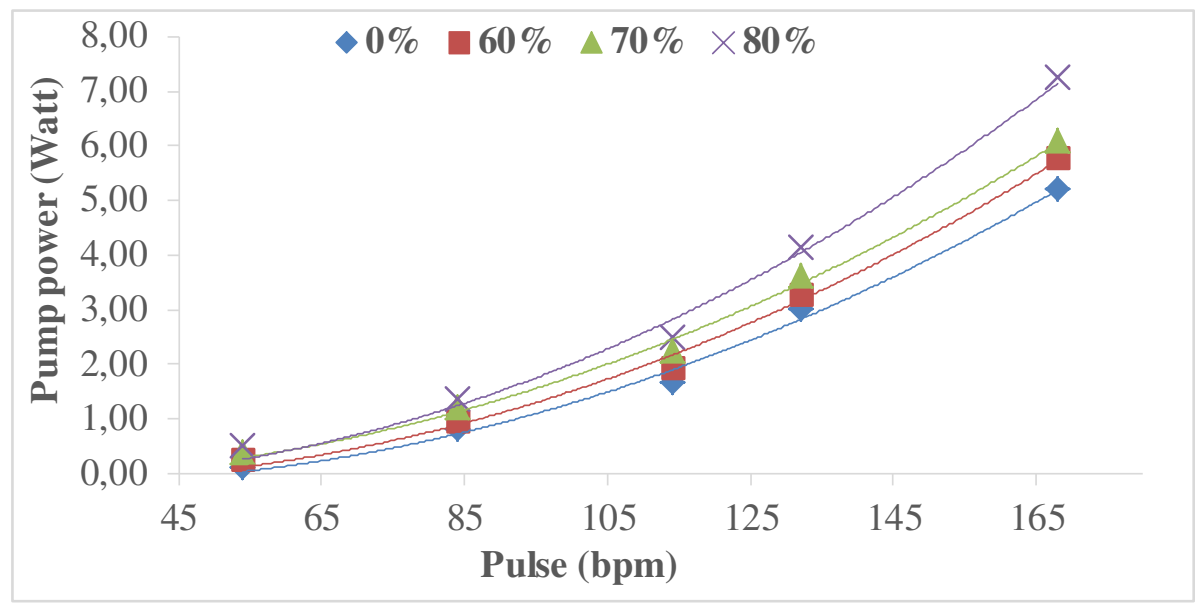

Figure 7. The effect of pulse values at different stenosis severities on the power consumed by the pump

In Figure 7, the effect of the pulse values in the peristaltic pump of the test area silicon pipe with areal rates of symmetrical stenosis at $0 \%, 60 \%, 70 \%$ and $80 \%$ on the power consumed by the pump is exhibited. In the figure, the amount of power consumed by the peristaltic pump increases concomitantly with the increasing pulse values. In addition, a gradual increase is observed in the amount of power spent on the pump in all pulse values ranging from a healthy artery with a stenosis rate of $0 \%$ to an artery with a stenosis rate of $80 \%$. The reason for this is that the pump will use more power to overcome the differential pressure caused by the narrowing of the pipe. This experimental data result establishes the necessity to likewise avoid activities requiring high pulse in human arteries with a high percentage of stenosis.

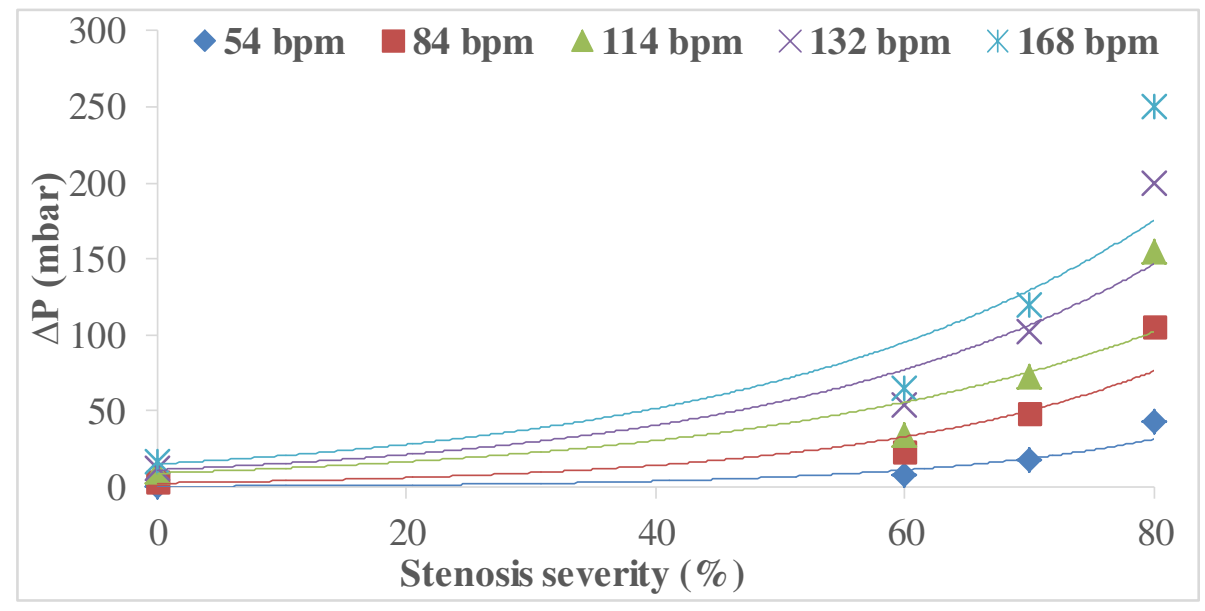

Figure 8. The effect of stenosis severity on pressure drop at different pulse values

In Figure 8, the effect of the test area location of the silicon pipe with certain areal rates of stenosis presenting the pulse values of $54,84,114,132$ and $168 \mathrm{bpm}$ on the pressure drop is 
represented. In the figure, $\Delta \mathrm{P}$ in the test area increases in accordance with the increasing stenosis severity. However, comparing the pulse values at the same stenosis severities, it was concluded that the $\Delta \mathrm{P}$ value increased as the pulse value increased. Furthermore, while the $\Delta \mathrm{P}$ values are close to each other at different pulse values for low stenosis severities, the $\Delta \mathrm{P}$ difference between these values increasingly grows with the increasing stenosis severity. This indicates that as the pulse increases in the vessels, $\Delta \mathrm{P}$ also grows, and this amount climbs even more with the increasing rate of stenosis [30].

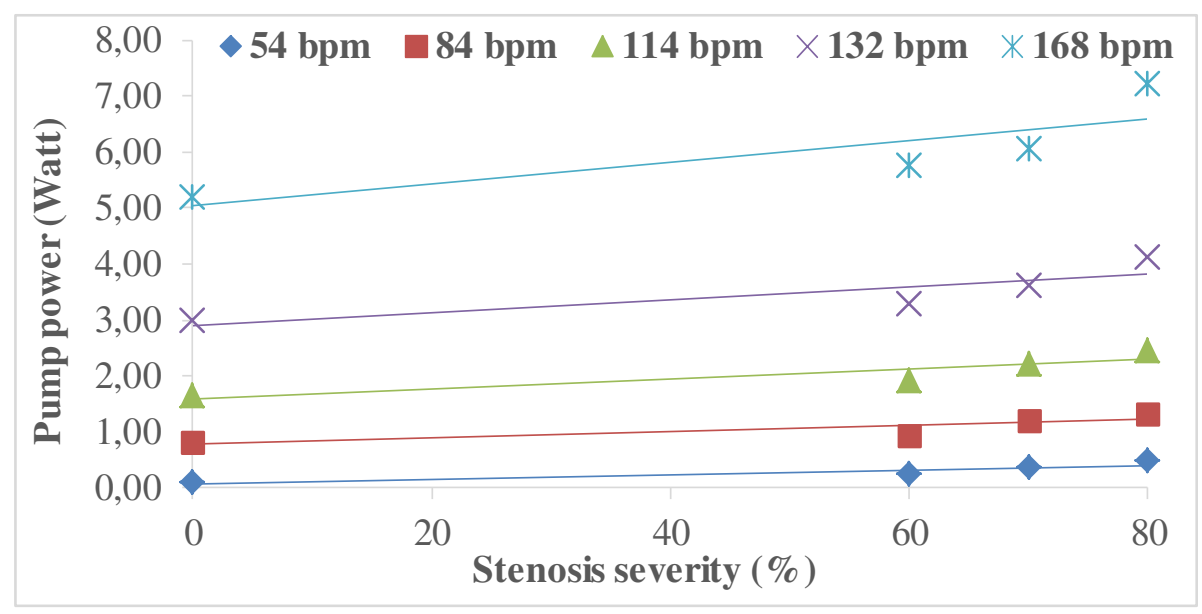

Figure 9. The effect of the stenosis severity on the power consumed by the pump at different pulse values

In Figure 9, the effect of the test area location of the silicon pipe with certain areal rates of stenosis presenting the pulse values of 54, 84, 114, 132 and $168 \mathrm{bpm}$ on the amount of power consumed in the peristaltic pump is exhibited. In the figure, the amount of power consumed in the pump in the test area increases with the increasing stenosis severity. Additionally, a gradual increase is observed in the amount of power spent on the pump in all pulse values ranging from a healthy artery, that is with a stenosis rate of $0 \%$, to an artery with a stenosis rate of $80 \%$. Furthermore, examining the slopes of the linear curves in each pulse value, the highest slope was observed at $168 \mathrm{bpm}$ and the lowest slope was $54 \mathrm{bpm}$. This increase in the pulse value in the artery with the same stenosis severity will cause the pump to consume more power than expected.

In Figure 10, the effect of the pulse values for 60 seconds in the test area silicon pipe with areal rates of symmetrical stenosis at $0 \%, 60 \%, 70 \%$ and $80 \%$ on the peristaltic pump outlet is represented. In the figures, it is observed that the difference between the maximum and minimum values of the pump outlet pressure grows with the increase of the pulse value for each stenosis severity level. Moreover, the maximum and minimum values of the pump outlet pressure increase as the rate of stenosis increases. 

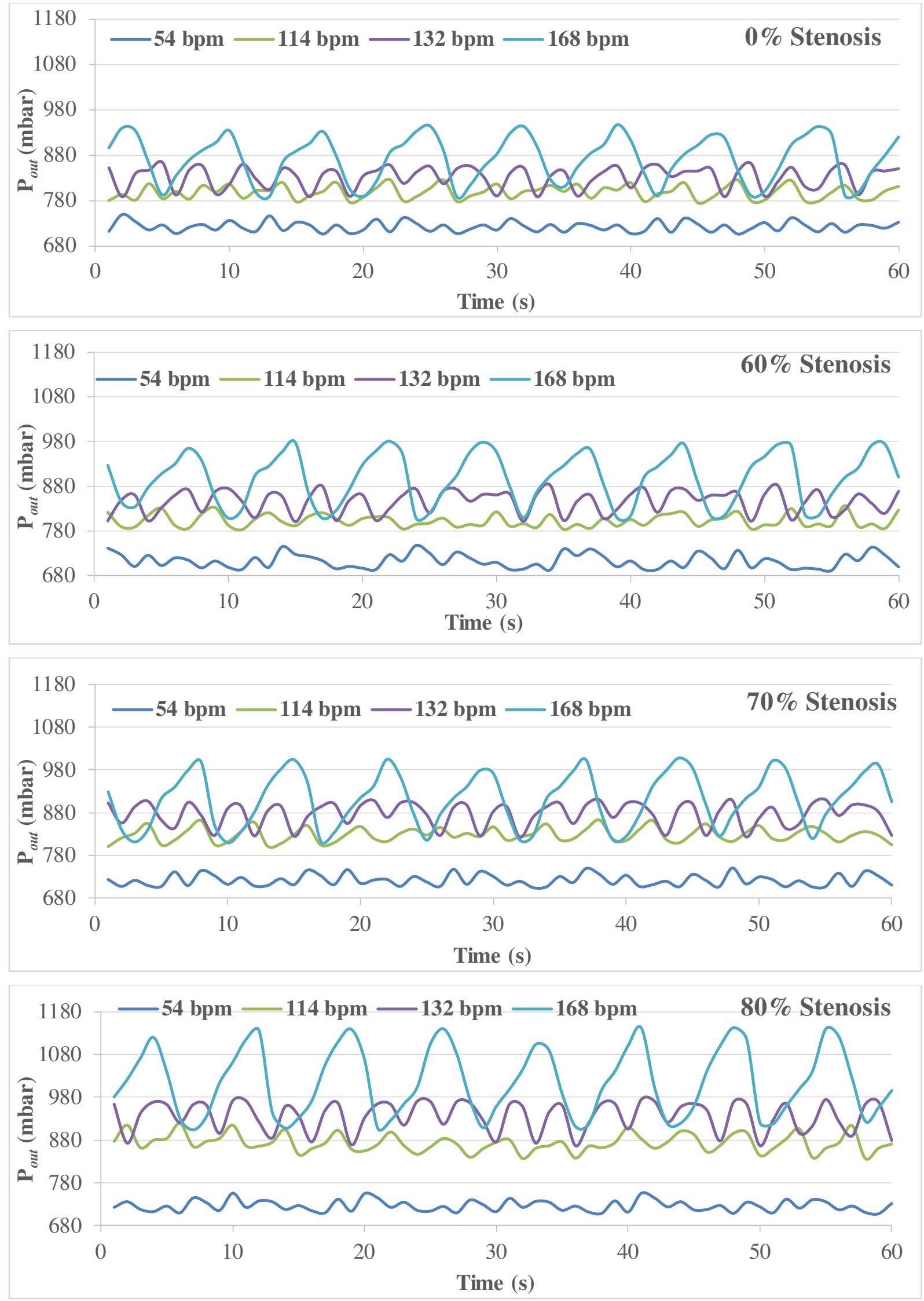

Figure 10. The variation of the pump outlet pressure over time at different pulse values 


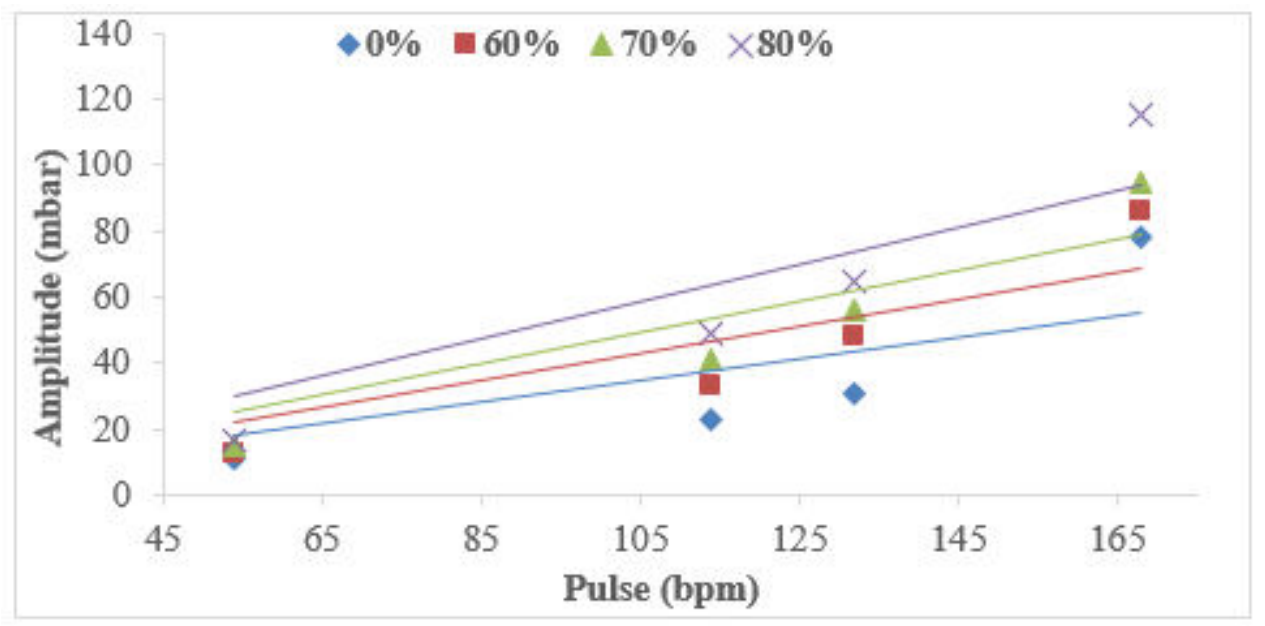

Figure 11. The effect of pulse values at different stenosis severities on differential pressure of pump

Figure 11 demonstrates the effects of the pulse values of the silicone pipe at different stenosis severities on the outlet pressure amplitude of pump. In the figure, in all stenosis severities, pump outlet pressures increase in accordance with the increase of pulse values because the increase of the pump flow rate and the subsequent increase of the pressure at the pump outlet in accordance with the increase in pulse values are expected [28], and this increase also led to an increase in the pump outlet pressure amplitude. In addition, a gradual increase is observed in the pump outlet pressure amplitude in accordance with the increase in the pulse values of pipes with stenosis severities ranging between $0 \%$ and $80 \%$.

\section{Conclusion}

In the experimental study, the hemodynamic properties of the vessel in different stenosis geometries were determined by setting up a test system similar to the heart mechanism, and the effect of the properties in these geometries on the peristaltic pump (heart) was investigated. The results are represented in items as follows:

- The increase in the pulse value elevates the pressure drop in the artery. For the same pulse values, the pressure drop increases in accordance with the increase in stenosis severity level, and the pressure drop reaches quite considerable values with the increase in the pulse value, particularly at stenosis severity levels greater than $70 \%$.

- Increase in flow elevates the pressure drop in the artery. At the same flow rates, the pressure drop climbs with the increasing severity of stenosis, and the pressure drop rises to critical values with the increase of flow at stenosis severities greater than $70 \%$.

- Pressure values at the peristaltic pump outlet, as expected, presented similar trends to the human arterial system. The pressure increases at the pump outlet in accordance with the increasing pulse value, and the pump outlet pressure elevates with the increasing severity of stenosis. 
- The pressure difference in the peristaltic pump grows concomitantly with the increasing pulse value. Furthermore, the differential pressure increased with the increase in stenosis severity.

- In the peristaltic pump, the power consumed by the pump increases in accordance with the increasing pulse value. On the other hand, at the same pulse value, the increasing severity of stenosis causes the pump to consume more power. This experimental data result reveals that, similarly, it is necessary to avoid activities requiring high pulse in human arteries with a high percentage of stenosis.

- Considering the amplitude values generated from the peristaltic pump outlet pressure data, low amplitude values occurred at low pulse values, and high amplitude values at high pulse values. Moreover, an increase was observed in the amplitude among the stenosis severities in accordance with the increase in the pulse value.

\section{Conflicts of Interest: None}

Funding: This study was sponsored by Atatürk University Scientific Research Projects Coordination Unit with the grant FCD-2019-7316.

Ethical Approval: Not required

\section{References}

[1] Chan, B.T., et al., Review on CFD simulation in heart with dilated cardiomyopathy and myocardial infarction, Computers in Biology and Medicine, 43 (2013), pp. 377-385

[2] Mozaffarian, D., et al., Heart disease and stroke statistics-2015 update: a report from the American Heart Association, Circulation, 131 (2015), pp. 29-322

[3] McElroy, M., et al., Left ventricular assist devices: Impact of flow ratios on the localization of cardiovascular diseases using computational fluid dynamic, Procedia CIRP, 49 (2016) pp. 163169

[4] Chatzizisis Y.S et al., Role of endothelial shear stress in the natural history of coronary atherosclerosis and vascular remodeling: molecular, cellular, and vascular behavior, Journal of the American College of Cardiology, 49 (2007), 25, pp. 2379-2393

[5] Lozano, R., et al., Global and regional mortality from 235 causes of death for 20 age groups in 1990 and 2010: a systematic analysis for the global burden of disease study 2010, Lancet, 380 (2012), pp. 2095-2128

[6] Ku, D.N., Blood flow in arteries, Annual Review of Fluid Mechanics, 29 (1997), 1, pp. 399-434

[7] Taylor, C.A., et al., Computational fluid dynamics applied to cardiac computed tomography for noninvasive quantification of fractional flow reserve: scientific basis, Journal of the American College of Cardiology, 61 (2013), 22, 2233-2241 
[8] Young, D.F., Tsai, F.Y., Flow Characteristics in Models of Arterial Stenoses-I. Steady Flow, Journal of Biomechanics, 6 (1973), 4, pp. 395-410

[9] Morgan, B.E., Young, D.F., An Integral Method for the Analysis of How in Arterial Stenoses, Bulletin of Mathematical Biology, 36 (1974), pp. 39-53

[10] Ahmed, S.A., Giddens, D.P., Velocity Measurements in Steady Flow Through Axisymmetric Stenoses at Moderate Reynolds Numbers, Journal of Biomechanics, 16 (1983), 7, pp. 505-516

[11] Jung, H., et al., Asymmetric Flows of Non-Newtonian Fluids in Symmetric Stenosed Artery, Korea-Australia Rheology Journal, 16 (2014), pp. 101-108

[12] Roy, M., et al., Modelling of Blood Flow in Stenosed Arteries, Procedia Computer Science, 115 (2017), pp. 821-830

[13] Gijsen, F.J.H., et al., The Influence of the Non-Newtonian Properties of Blood on the Flow in Large Arteries: Steady Flow in a Carotid Bifurcation Model, Journal of Biomechanics, 32 (1999), pp. 601-608

[14] Park, S.M., et al., In Vitro Hemodynamic Study on the Stenotic Right Coronary Artery Using Experimental and Numerical Analysis, Journal of Mechanics in Medicine and Biology, 10 (2010), 4, pp. 695-712

[15] Anastasiou, A.D., et al., Experimental Investigation of the Flow of a Blood Analogue Fluid in a Replica of a Bifurcated Small Artery, Medical Engineering \& Physics, 34 (2012), 2, pp. 211-218

[16] Husain, I., et al., Three Dimensional Pulsatile Non-Newtonian Flow in a Stenotic Vessel, Computational Problems in Engineering (Ed. Mastorakis, N., Mladenov, V.,), Springer International Publishing, Switzerland, 2014, pp. 55-64

[17] Apostolidis, A.J., et al., Non-Newtonian Effects in Simulations of Coronary Arterial Blood Flow, Journal of Non-Newtonian Fluid Mechanics, 233 (2016), pp. 155-165

[18] Back, L.H., et al., Measurement and Prediction of Flow Through a Replica Segment of a Mildly Atherosclerotic Coronary Artery of Man, Journal of Biomechanics, 19 (1986), 1, pp. 1-17

[19] Vesely, O., et al., Experimental Study of Effect of Stenosis Geometry on Flow Parameters, EPJ Web of Conferences, 92 (2015), 02105

[20] Novakova, L., et al., Vascular Stenosis Asymmetry Influences Considerably Pressure Gradient and Flow Volume, Physiological Research, 65 (2016), pp. 63-69

[21] Kamangar, S., et al., The Influence of Geometrical Shapes of Stenosis on the Blood Flow in Stenosed Artery, Sains Malaysiana, 46 (2017), 10, pp. 1923-1933

[22] Ahmed, S.A., Giddens, D.P., Pulsatile Poststenotic Flow Studies with Laser Doppler Anemometry, Journal of Biomechanics, 17 (1984), 9, pp. 695-705

[23] Siouffi, M., et al., The Effect of Unsteadiness on the Flow Through Stenoses and Bifurcations, Journal of Biomechanics, 17 (1984), 5, pp. 299- 315

[24] Rabby, M.G., et al., Pulsatile Non-Newtonian Blood Flow through a Model of Arterial Stenosis, Procedia Engineering, 56 (2013), pp. 225-231 
[25] Beratlis, N., et al., A Numerical and Experimental Investigation of Transitional Pulsatile Flow in a Stenosed Channel, Journal of Biomechanical Engineering, 127 (2005), 7, pp. 1147-1157

[26] Liou, T.M., Liou S.N., A review on in vitro studies of hemodynamic characteristics in terminal and lateral aneurysmmodels, Proc Natl Sci Counc Repub China (B), 23 (1999), 4, pp. 133-148

[27] Leuprecht, A., Perktold, K., Computer simulation of non-Newtonian effects on blood flow in large arteries, Comput Methods Biomech Biomed Engin, 4 (2001), pp. 149-163

[28] Cengel, A.Y., Cimbala, J.M., Fluid Mechanics Fundamentals and Applications, McGraw-Hill Education, New York, USA, 2014

[29] Hoskins, P.R., Lawford, P.V., Cardiovascular Biomechanics, Atherosclerosis (Ed. Hoskins, P.R., et al.) Springer International Publishing, Switzerland, 2017, pp. 285-306

[30] Banerjee, R.K., et al., Physiological flow analysis in significant human coronary artery stenoses, Biorheology, 40 (2003), 4, pp. 451-476

[31] Pandey, R., et al., Numerical computation of blood hemodynamic through constricted human left coronary artery: Pulsatile simulations, Computer Methods and Programs in Biomedicine, 197 (2020), 105661

[32] Verde,W.M., et al., Experimental investigation of pressure drop in failed Electrical Submersible Pump (ESP) under liquid single-phase and gas-liquid two-phase flow, Journal of Petroleum Science and Engineering, 198 (2021) 108127 